\title{
Feminismo y ecología: el rol de las universidades frente a la crisis ambiental ${ }^{1}$
}

\section{Ana Victoria Portocarrero*}

Berta Cáceres fue asesinada el día 3 de marzo en su casa de habitación. Cuatro balazos le arrebataron la vida a esta indígena lenca hondureña, quien alzó la voz a favor de los ríos y bosques, y presentó resistencia pacífica pero constante a las pretensiones del gobierno hondureño y de empresas nacionales y transnacionales, de desarrollar megaproyectos de "desarrollo" a costa de la vida natural y de los derechos de los pueblos indígenas.

Tres elementos se juntan en la historia de Berta: primero, era una mujer; segundo, era una indígena; y tercero, era una luchadora social en favor del medio ambiente y de los derechos de los pueblos indígenas. Tres elementos que no casualmente la llevaron a la muerte en un mundo que extermina a todo aquel o aquella que se rebele contra las cadenas del desarrollismo, el capitalismo, el racismo y el patriarcado.

Para entender la relación entre feminismo y ecología, y para identificar el rol de las universidades y los y las académicas en la búsqueda de soluciones reales a la crisis ambiental y sistémica que vivimos, no hay mejor ejemplo que conocer la vida y la lucha de Berta Cáceres.

\section{¿Por qué luchaba Berta y sigue luchando hoy día el pueblo lenca?}

Después del golpe militar en Honduras en 2009, el gobierno otorgó concesiones para construir 47 represas con el fin de impulsar centenares de nuevos proyectos mineros. Una empresa china se unió con una compañía hondureña para construir una represa en el río Gualcarque o río Blanco, que afectaría a cientos de

Ponencia presentada el Día Internacional de las Mujeres en la Universidad Nacional de Costa Rica el 8 de marzo de 2016.

Programa Interdisciplinario de Estudios de Género, Facultad de Humanidades y Comunicación, Universidad Centroamericana, Managua. Correo electrónico: aportocarrero@ns.uca.edu.ni 
habitantes del pueblo lenca.

De acuerdo a Berta Cáceres, esta concesión violaba convenios relacionados a los derechos de los pueblos indígenas, y fue hecha sin ningún tipo de consideración hacia los pobladores y custodios del río. En dos cabildos abiertos y en más de 150 asambleas indígenas la población decidió que no quería el proyecto, no quería daños al río, y no quería su privatización. Pero la voz del pueblo no jugó un papel importante en las negociaciones que se hicieron entre el gobierno y la empresa privada. "Hicimos denuncia" dijo Berta, pero "nadie ha escuchado a este pueblo".

La resistencia del pueblo lenca desde ese momento les ha costado no solo la reciente muerte de Berta Cáceres, sino de otros líderes lencas como Tomás García, a quien el ejército hondureño disparó a matar el 14 de julio de 2013 durante una protesta pacífica.

\section{¿De qué se trata la protesta de este pueblo y de esta mujer?}

Es una protesta que va mucho más allá del amor al río Gualcarque. Es una protesta ante un sistema que intenta de manera sistemática imponer un modelo de desarrollo con el que no todas y todos estamos de acuerdo, y en particular no están de acuerdo los pueblos indígenas.

El modelo de desarrollo hegemónico extractivista promueve una mirada de la naturaleza utilitarista en función de la generación de beneficios económicos que son acumulados por grupos particulares en detrimento no solo de otros grupos sociales - en este caso indígenas - sino además del equilibrio ambiental. No es ninguna novedad que los supuestos beneficios del "desarrollo" han probado no distribuirse de manera equitativa entre las personas, y no es tampoco ninguna novedad que el modelo de desarrollo al que llamamos actualmente "progreso" ha acabado con la naturaleza y con los medios de vida de las poblaciones indígenas y campesinas. Sin embargo este reclamo va incluso más allá de la crítica al desarrollismo hegemónico, en crisis ya dese al menos los años 90. Esta crítica incluso cuestiona modelos más "sostenibles" de desarrollo, con los que nos sentimos más cómodos y cómodas a veces, pero que siguen viendo el mundo natural de manera utilitaria.

Des Gasper, Asunción Lera St. Clair y mi persona realizamos entre 2011 y 2012 una serie de análisis de los discursos dominantes sobre medio ambiente, cambio climático y desarrollo de organizaciones internacionales tan importantes como el Banco Mundial y el Programa de Naciones Unidas para el Desarrollo. En nuestro trabajo analizamos informes producidos por ambas instituciones relativos a la problemática del cambio climático y su relación con los modelos de desarrollo promovidos en el mundo y particularmente en el mundo en desarrollo, y encontramos una serie de hallazgos interesantes.

Nuestro primer hallazgo fue que existía una diferencia importante en el diagnóstico de la problemática que hacía el Banco Mundial y la que hacía el PNUD. Por ejemplo, mientras el BM se centraba más en los costos económicos que el fenómeno del cambio climático implica para el desarrollo de los países, y en encontrar maneras de aminorar lo más posible dichos costos desde una perspectiva meramente tecnocrática, el PNUD contemplaba los problemas de justicia social y 
éticos asociados al cambio climático, señalando por ejemplo que detrás de la crisis ambiental se encuentra la crisis del modelo de desarrollo basado en el consumo y la producción siempre en crecimiento, que las poblaciones que más reciben los efectos del cambio climático son las que menos han sido responsables del mismo, y que por ende es una cuestión de justicia social que las soluciones al problema contemplen no solo la correcta reparación hacia las poblaciones más impactadas, sino además un cuestionamiento profundo al modelo de desarrollo implementado en los países desarrollados, que ha desembocado en la crisis ambiental.

Ese necesario cuestionamiento, sin embargo, se ve opacado por nuestro segundo y más importante hallazgo. En las propuestas de solución a la problemática, curiosamente ambas instituciones tenían más coincidencias que diferencias. En síntesis, las propuestas podrían resumirse de la siguiente manera:

(1) los países en desarrollo necesitan un mayor desarrollo; (2) las economías de altos ingresos deben adoptar un modelo que ponga precio al carbono: ya sea un impuesto al carbono o un sistema de límites máximos y comercio; (3) estos mecanismos van a generar los fondos que deben ser administrados por un mecanismo multilateral para la transferencia de recursos financieros y de tecnología a los $\mathrm{PMD}^{2}$ tanto para la mitigación como para la adaptación; (4) las transferencias serán condicionadas a la implementación de programas y asunción de compromisos por parte de los PMD relacionados con objetivos cuantitativos y cambios de política en mitigación, adaptación y gestión de recursos como el agua, la tierra y la energía; y (5) una mayor dependencia y confianza en los mercados competitivos (Gasper et al., 2013b, p. 34).

El punto central de nuestro hallazgo es que a pesar de que el PNUD cuestiona en un primer momento la lógica de crecimiento y consumo latente en el modelo de desarrollo que produjo el problema ambiental que vivimos, la propuesta de solución es más desarrollo, con modificaciones, pero siempre basado en una lógica neoliberal, en donde son los criterios del mercado, y no una ética del cuidado y del equilibrio, los que determinan quiénes pueden contaminar, a qué precio y en qué cantidad. Los mercados deben ser más y más "libres", sugieren los informes, a fin de no interferir en la movilización de bienes vitales como son la alimentación y el agua. Dichos bienes vitales, además, deben ser privatizados para asegurar en teoría, su mejor y más eficiente uso. Y son las poblaciones con mayores recursos las que al final tendrán acceso a los mismos. En esta lógica neoliberal, la naturaleza no es más que un recurso que debemos aprender a usar de manera más eficiente.

En un artículo publicado en 2013 sostengo que esta lógica detrás de las soluciones a la problemática ambiental no hace más que sostener y justificar el mismo modelo de relaciones económicas y comerciales internacionales, en donde los países menos desarrollados se vuelven el escenario en el cual se debe actuar, no con sus propios recursos humanos, intelectuales, culturales y tecnológicos, sino con recursos provenientes de instituciones financieras internacionales y con tecnología importada, a cambio claro está, de una gestión de los recursos naturales condicionada a favor de los intereses de grupos económicos y potencias mundiales particulares. Sostengo en dicho artículo, que las coincidencias entre este modelo 
de acción y las medidas de ajuste estructural implementadas en los países menos desarrollados desde los años 90, no son mera coincidencia.

La lucha del pueblo lenca parte de una cosmovisión distinta. En ésta, el mundo natural no es un recurso para el bienestar humano sino parte integral de su cultura e identidad. El desarrollo, por ende, tanto en su versión más cruda como en su versión alternativa, no tiene sentido alguno en tanto en ambos casos parte de una idea dual sobre la relación entre el ser humano y el mundo natural. Alterar la vida del río Gualcarque, por lo tanto, no solo implica la desaparición de los medios de vida de las poblaciones aledañas al mismo, sino la destrucción de la identidad y cultura de estos grupos poblacionales.

Es interesante en esta lectura de la resistencia lenca, que a Berta Cáceres la acusaron en algún momento de interponerse al progreso y al desarrollo sostenible, y de vender una imagen negativa del gobierno hondureño, llegando incluso a ponerle demandas por sus actos de resistencia a un modelo de desarrollo que nunca fue consultado con las comunidades indígenas.

Me gustaría ahora pasar a un segundo tema, pues la persona que fue asesinada no era cualquier persona, era además de una activista medioambiental, una mujer, una indígena, y una feminista. Nos pregunto, ¿es casual que la voz de los ríos y los bosques fuera la de una mujer indígena feminista? En absoluto. Desde al menos los años 70 se viene identificando dentro del mundo académico y del activismo, la relación entre feminismo y ecología, y más recientemente la relación de ambos temas con el racismo y la exclusión de clase. A continuación presento algunas de las perspectivas más relevantes sobre estos temas.

Dentro de las teóricas de la primera ola del ecofeminismo, primer término que se usó para analizar la relación entre feminismo y ecología, se encuentra a la feminista francesa Francoise d'Eaubonne que a principios de los años 70 empezó a teorizar la relación entre la superpoblación, la devastación de la naturaleza y la dominación masculina. De acuerdo a d'Eaubonne, para resolver la producción superflua y la devastación de la naturaleza, habría que cuestionar la relación entre los sexos, y el poder sobre la reproducción que ha sido arrebatado a las mujeres. Pero ella va más allá, señalando que debe cuestionarse el modelo de civilización falocéntrico tanto capitalista como socialista, por ser ambos insostenibles ecológicamente.

Ideas similares promovió la filósofa feminista estadounidense Mary Daly, quien recuperó en su abordaje de la relación entre opresión de género y devastación de la naturaleza, el dualismo generizado: Naturaleza / Cultura, en donde la naturaleza es adjudicada a las mujeres, y la cultura a los hombres, dándole más valor a la cultura que al mundo natural. Para ecofeministas como Daly, el ejercicio que había que hacer era el de revertir los valores y darle superioridad a la Naturaleza sobre la Cultura, en tanto la última se ha basado en la destrucción de formas de vida y en la opresión de las mujeres.

Más recientemente, ecofeministas como Vandana Shiva han explorado ideas que han venido a englobarse como "ecofeminismo espiritualista". De acuerdo a la cosmología hindú recogida por esta científica India en sus análisis, Shakti y Purusha, la energía femenina y masculina, se manifiestan en armonía en la naturaleza (Prakriti). Lo que ha sucedido, según Shiva, es que el mecanismo reduccionista de la 
Modernidad Occidental, para la cual la naturaleza se convierte en mera materia prima para la producción, ha destruido el principio femenino, desbalanceando Prakriti, y afectando de manera más significativa a las poblaciones más marginalizadas, entre ellas a mujeres y niñas rurales.

El "mal desarrollo", como le llama Shiva, exportado por el colonialismo actual, consiste en la imposición del modelo occidental a todas las culturas, tanto en la India como en Honduras, Costa Rica o Nicaragua. Este mal desarrollo tiene impactos diferenciados de género, con una carga significativa en las mujeres. Para ilustrar:

Con el fenómeno de los monocultivos en favor de la exportación, los antiguos trabajos de las mujeres y sus conocimientos de la vegetación del lugar son despreciados e invisibilizados en favor de los conocimientos asociados con el modelo de desarrollo hegemónico. A estos saberes, tienen más acceso los hombres, quienes en general son los dueños de las tierras, toman decisiones sobre las mismas, y tienen acceso a capacitaciones técnicas y créditos.

La deforestación obliga a las mujeres y a las niñas a caminar kilómetros en busca de leña y agua. Son ellas quienes se encargan de estas actividades, y esto afecta el tiempo para otras tareas, para el descanso, para la escuela, y además las pone en un mayor riesgo de violaciones en los caminos.

El "progreso" empobrece a las mujeres al eliminar toda posible ganancia en sus tareas artesanales colaterales y las coloca en una situación de mayor dependencia al hombre, a quienes les conceden los créditos y programas de ayuda para las actividades que en el nombre del "desarrollo" se consideran importantes.

Lo que se denomina desarrollo, considera Shiva, es violencia hacia las mujeres y la naturaleza, y tiene su origen en los postulados patriarcales de homogeneidad, dominación y centralización que constituye el fundamento de los modelos de pensamiento y estrategias de desarrollo dominantes. Por ende, la muerte de Prakriti y la crisis ecológica es, en su raíz, la muerte del principio femenino, considera.

Val Plummwood, Ynestra King, Barbara Holland Cunz, y Karen Warren también ecofeministas, se alejan un poco de los postulados a su juicio esencialista de la primera ola del ecofeminismo, y proponen una mirada más constructivista de la relación entre opresión de las mujeres y degradación de la naturaleza. Para ellas, la lógica del dominio no es esencialmente masculina, sino tan solo históricamente masculina. Es decir, tiene que ver con la construcción social del género, más que con una esencia.

Por su parte en latinoamérica el ecofeminismo ha tenido una relación histórica con la teología de la liberación. Entre las principales exponentes tenemos a Ivonne Gebara, quien propone que "una articulación íntima entre una línea feminista de pensar la vida y una línea ecológica, nos abre no sólo una posibilidad real de igualdad entre mujeres y hombres de diferentes culturas, sino una relación diferente entre nosotros, con la tierra y con todo el cosmos".

Dos características propias tiene la propuesta latinoamericana: por un lado la revalorización de las cosmovisiones autóctonas (antiguas) y por otro el énfasis en la praxis de liberación. En otras palabras, de nada nos sirve la teorización excesiva 
entre feminismo y ecología, si no nos lleva a acciones concretas para parar tanto la destrucción masiva del ecosistema como la mejora de la vida de las mujeres.

Las miradas ecofeministas han sido retomadas en ciertos aspectos y cuestionadas en otros por propuestas ambientalistas feministas como las de Bina Agarwal, también de la India. Agarwal señala que algunas propuestas ecofeministas pueden ser peligrosas por esencialistas y por poner mayor énfasis en los aspectos ideológicos o simbólicos de la relación entre género y medio ambiente que en los aspectos materiales. En este sentido, ella propone poner atención a los elementos materiales de dicha relación, pero también la reivindicación del papel activo de las mujeres en la búsqueda de alternativas y resistencias, y no solo de su rol como víctimas de los daños ambientales.

De acuerdo a Agarwal, la existencia de una división del trabajo y una distribución de la propiedad y del poder mediada por el género, la clase, y la etnia, estructura las interacciones de las personas con la naturaleza y, en consecuencia, los efectos del cambio ecológico sobre las personas así como sus respuestas a estos cambios.

El vínculo de las mujeres con la naturaleza las sitúa como víctimas de la degradación ambiental, pero también como depositarias de un saber sobre ésta que no tienen los hombres y que debe formar parte de las estrategias de resistencia, propone. En otras palabras, en la búsqueda de mayor justicia de género y ecológica, deben combatirse a los grupos dominantes que concentran la propiedad, el poder y el privilegio de controlar los recursos, a la vez que se combata a los grupos que determinan las formas de pensar, es decir, aquellos que controlan la enseñanza, los medios de comunicación y otras instituciones difusoras de ideas.

Aquí empezamos a abordar dos temas relevantes. Primero, tocar el orden de género y el orden ambiental es tocar el poder. Y segundo, tocar dicho poder es cuestionar el sistema de pensamientos que consideramos válido, legítimo, importante. Es en este tema, particularmente, donde nos vemos implicadas directamente como universidades.

Nos centramos, entonces, desde un marco teórico que politiza la ecología en su relación con el feminismo y que con pensadoras como Dianne Rocheleau, analizamos los contextos en donde la raza, la clase, la cultura y la identidad nacional interactúan con el género para conformar nuestras experiencias de «el ambiente» e intereses en el mismo, utilizando las críticas feministas de la ciencia y los análisis y acciones de los movimientos feministas y ambientales como puntos de partida.

Desde la ecología política feminista, por tanto, se nos insta a proponer una ciencia transformadora y socialmente justa, que parta desde la interseccionalidad de opresiones y privilegios de las personas, y sobre todo que reconozca y combine los conocimientos localizados, con los saberes tradicionalmente considerados como legítimos dentro de las universidades.

Me gustaría ahora entrar a la sección conclusiva de esta charla, volviendo a conectar con Berta, ya no solo como la mujer, la indígena, la activista ambiental, sino sobre todo como una episteme. A Berta la llamaban la madre de todos los ríos por su incansable lucha en defensa del río Gualcarque. Para ella, y para la comunidad lenca, el río tiene una importancia ancestral espiritual porque desde su cosmovisión 
en éste vive el espíritu femenino. "Siempre se nos ha enseñado de que son las niñas las que custodian los ríos”, dijo en alguna ocasión, enfatizando que además, el río representa una fuente de alimentación y salud para las y los indígenas, por las plantas medicinales a su alrededor, así como una fuente de agua para las poblaciones cercanas a éste. "El río", dice Berta, "significa vida".

Pero, ¿por qué es importante el significado del río? Porque si seguimos planteando soluciones a la crisis ambiental solamente desde los significados de una forma particular de ver el mundo, estamos consciente o inconscientemente generando violencia. El río puede significar para instituciones de desarrollo solamente una fuente de alimentación, o de energía, puede significar un elemento para disminuir la pobreza o incluso para fomentar energía renovable. Siendo así, el río puede ser modificado si dicha modificación se justifica en un cálculo de costos versus beneficios. Pero, ¿cómo calculamos el costo de la pérdida de una cultura? ¿Cómo se puede sacar el beneficio o el costo de la vida del río? Y si queremos realmente considerar las cosmovisiones y derechos de las poblaciones indígenas, ¿de qué manera podemos calcular si es o no rentable prescindir del espíritu femenino que se adjudica a las fuentes de agua?

Estamos entonces frente a una lucha epistémica que no debemos dejar librar únicamente a las poblaciones indígenas o grupos de ambientalistas en general. Estamos frente a una lucha epistémica que toca el centro de nuestro quehacer como universidades, que es la producción de conocimientos que permitan transformar nuestras sociedades en lugares más habitables, justos, en donde la vida sea posible para todos y todas, y no solo para unos pocos.

Bina Agarwal señala que lo que está en juego no es la ciencia moderna en sí, sino el proceso por el cual se genera y se aplica lo que se considera "conocimiento científico». De acuerdo a ella y otras pensadoras y pensadores en esta línea, debemos partir por cuestionar las jerarquías entre trabajo intelectual y físico, ciudad y campo, varones y mujeres, y promover búsqueda de soluciones a la crisis ambiental que partan de lo que Boaventura de Sousa Santos llama, una "ecología de saberes". En esta ecología de saberes no se estaría descartando el conocimiento científico, sino que se pondría en diálogo con los saberes ancestrales, y con los saberes situados de las poblaciones históricamente marginadas de los mundos académicos, siendo especialmente relevante en este caso, el conocimiento de las mujeres rurales, de las campesinas, de las indígenas. Dada su vinculación en el día a día con la naturaleza, sus prácticas y puntos de vista son relevantes para la búsqueda de verdaderas alternativas.

La búsqueda de soluciones deben involucrar, por ende, cambios sustanciales en la composición de lo que se produce; las tecnologías usadas para producir; los procesos por los que se llega a decidir qué producir y cómo; los sistemas de conocimiento en los que se basan dichas decisiones; y la distribución por clase, etnia y género de las responsabilidades y tareas relacionadas con el medio ambiente. Se requiere un ensanchamiento de la definición de "ciencia» para que abarque múltiples fuentes de saber e innovación, y con ello múltiples modos de relación humana y ecológica.

Para Vandana Shiva el conocimiento que tienen las mujeres del mundo natural, que ha sido sistemáticamente marginado por la ciencia reduccionista 
moderna y los paradigmas de desarrollo, es vital no solo para lograr su propio sustento, sino para realmente comprender las complejas interconexiones entre vida humana y vida natural, producción y reproducción, lo cual debe ser un punto clave a considerar en las acciones para mitigar la crisis ambiental. Por su parte, añade Rocehelau, el conocimiento de las mujeres debido a los roles de género establecidos y a su cercanía a la naturaleza, contiene habilidades de integración y de manejos de sistemas complejos que son opuestas a las ciencias especializadas y al conocimiento fragmentado que se ocupan solo de un dominio particular. Este pensamiento integral, interconexo, holístico, por lo general informal y no especializado necesariamente, debe ser parte importante en los diálogos sobre estos temas.

El último post de Berta Cáceres en facebook, el día 1 de marzo a las 5.51 de la tarde, solamente 32 horas antes de ser asesinada, fue sobre un foro sobre energías alternativas desde la visión indígena del COPINH, el Consejo Cívico de Organizaciones Populares e Indígenas de Honduras. El foro anunciado para los días 2, 3 y 4 de marzo en el centro de capacitación Utopía, tenía como consigna: "Energías alternativas fuera de la lógica consumista, privatizadora, y depredadora de la vida”. Proponer ideas fuera de esa lógica tan dominante incluso en nuestros espacios universitarios, desafortunadamente, le ha implicado al pueblo lenca poner en riesgo la vida, y perderla en el caso de Berta. El asesinato de Berta es particularmente doloroso pues con esto se pretende matar más que a una persona, se pretende exterminar ideas y cosmovisiones que se resisten a esta lógica totalizante. Resuenan en mis oídos las palabras de Boaventura, que incesantemente nos previene que de la mano de los genocidios se encuentran los epistemicidios.

"Cuando iniciamos la lucha por río Blanco me metí al río. Podía hablar con el río, sentía lo que el río me decía, y yo sabía lo duro que iba a ser. Pero sabía que íbamos a triunfar. El río me lo dijo", dijo Berta tras lograr, junto con el resto del pueblo organizado, que la empresa china y el Banco Mundial se retiraran del proyecto al menos momentáneamente. El río quizá también le dijo a Berta que su vida estaba en juego, que la lucha no terminaba ahí, y Berta a pesar de esto decidió seguir adelante.

"No es un crimen defender nuestros derechos como pueblos indígenas", les dijo en alguna ocasión a los y las lencas, y es esa reivindicación la que tenemos que asumir las universidades sin miramientos frente a este tremendo reto ambiental y humano que vivimos. 